Article

\title{
Electromagnetically Induced Transparency in Symmetric Planar Metamaterial at THz Wavelengths
}

\author{
Abdelwaheb Ourir ${ }^{1, *}$, Bruno Gallas ${ }^{2}$, Loic Becerra ${ }^{2}$, Julien de Rosny ${ }^{1}$ and \\ Pierre Richard Dahoo ${ }^{3}$
}

1 Laboratoire Ondes et Acoustique (LOA), Institut Langevin, ESPCI ParisTech, UMR 7587, CNRS, 1 rue Jussieu, Paris 75005, France; E-Mail: julien.derosny@espci.fr

2 Institut des NanoSciences de Paris, UMR 7588, CNRS-UPMC, 4 place Jussieu, 75252 Paris cedex 05, France; E-Mails: bruno.gallas@insp.jussieu.fr (B.G.); loic.becerra@insp.jussieu.fr (L.B.)

3 LATMOS, Université de Versailles-Saint-Quentin-en-Yvelines, CNRS-UPMC-UVSQ (UMR 8190), Guyancourt 78280, France; E-Mail: Pierre-Richard.Dahoo@latmos.ipsl.fr

* Author to whom correspondence should be addressed; E-Mail: a.ourir@espci.fr;

Tel.: +33-1-80-96-30-39.

Received: 18 February 2015 / Accepted: 4 March 2015 / Published: 19 March 2015

\begin{abstract}
We report the experimental observation and the evidence of the analogue of electromagnetically-induced transparency (EIT) in a symmetric planar metamaterial. This effect has been obtained in the $\mathrm{THz}$ range thanks to a destructive Fano-interference between the two first modes of an array of multi-gap split ring resonators deposited on a silicon substrate. This structure is a planar thin film material with four-fold symmetry. Thanks to this property, a polarization-independent transmission has been achieved. The proposed metamaterial is well adapted to variety of slow-light applications in the infrared and optical range.
\end{abstract}

Keywords: metamaterial; induced transparency; slow light; infrared

\section{Introduction}

Electromagnetically induced transparency (EIT) is an optical phenomenon that is characterized by the elimination of the effect of a medium on a propagating beam of electromagnetic radiation in a specific frequency band [1]. This phenomenon has received much attention due to its interesting physics [1-3] and important applications such as the transfer of quantum correlations [4], nonlinear 
optical processes at low light levels, and slow light propagation [5,6]. In 2008, EIT in metamaterial has been demonstrated by a coupled radiative-dark plasmonic mechanism [7]. In this case, EIT was obtained in resonators, or coupled resonators, where two resonant modes with different symmetries could be led to interfere at the same frequency range hereby inducing a band of transparency. Tunable transparency and slow light behavior have been also demonstrated by using a thermally active superconductor-metal coupled resonator on a sapphire substrate [8]. Recently, an analogous behavior of EIT, denoted as phonon-induced transparency (PIT), has been observed in stacked bilayer graphene nanoribbons [9].

Generally, a broken symmetry is used as a prerequisite for plasmonic EIT, since only the asymmetry of the resonators should allow the excitation of the forbidden dark mode [7-11]. As a result, these structures show polarization dependent response. Nevertheless, plasmonic EIT in symmetric structures has been numerically demonstrated by enabling plasmonic modes beyond the fundamental ones [12]. Generation of the plasmonic analogue of EIT from the second order plasmon resonance has been proposed for stacked optical metamaterials [13]. However, the alignment of the resonators of the different layers makes their fabrication a tough task. Recently, an effect reminiscent of EIT has been observed in planar metamaterial $[14,15]$. These effects were obtained by coupling two different types of resonators placed in the same plane. Even though this type of planar structure simplifies noticeably the fabrication in the infrared and visible range, it remains distinctly polarization dependent.

In this work, we propose to achieve an EIT-like effect in a symmetric planar metamaterial. The metamaterial we investigate, consists of an array of multi-gap split ring resonators (MSRRs) deposited on a thick silicon substrate. We show that an EIT-like transmission band can be obtained in the $\mathrm{THz}$ range thanks to a destructive interference between modes of different orders. We study the effect of the substrate on the dipole resonance and on the higher order plasmon resonances of this planar metamaterial. We demonstrate numerically and experimentally that a high group index can be achieved with this metamaterial for both vertical and horizontal polarizations in a narrow $\mathrm{THz}$ spectral range.

\section{EIT in Symmetric Planar Metamaterial}

The first EIT-like optical properties have been realized using a coupled radiative-dark plasmonic mechanism [7]. A simple metal strip and two parallel metal strips with a small separation have been used to generate the radiative and the dark mode respectively. Here we propose to design different sub-atoms in order to achieve an EIT-like effect in a structure showing four-fold symmetry. We start by performing numerical study using finite difference time domain (FDTD) calculations (CST Software) to investigate the response of different structures under a linearly polarized wave. First we consider a pair of metallic stripes parallel to the electric field. This sub-atom may act as an optical dipole antenna. The stripes are made of 80 -nm-thick and $1.4-\mu \mathrm{m}$-wide gold material. Figure 1a shows the considered structure and the electric field amplitude calculated near one of the metallic strip ends as a function of the frequency for a normal incidence. In this configuration, this radiative dipole mode could serve as the bright mode in an EIT-like plasmonic system. For a pair of metallic stripes orthogonal to the electric field, a normal incidence does not excite the first mode of the structure. In order to identify potential dark modes in this configuration, an incidence of $45 \mathrm{deg}$ is used as in ref [7]. 
Figure $1 \mathrm{~b}$ shows the electric field amplitude as a function of the frequency for this incidence. A resonant mode appears in this configuration. This mode is as a quadrupole dark mode with high quality factor.

The realization of dipole-quadrupole coupling is the key to obtaining EIT-like effect. Therefore, the combination of the two proposed sub-atoms should produce this type of effect. In order to confirm this hypothesis, we consider the structure of Figure 1c. The unit cell of the obtained metamaterial is a square-shaped SRR with 8 metallic gaps. The lattice of the unit cell is $10 \mu \mathrm{m}$. Figure $1 \mathrm{~d}$ shows the transmittance and the reflection of the obtained structure. A broadband first resonance followed by a sharp resonance are observed. These two resonances are located on the positions of the dipolar and quadrupolar modes identified previously. When illuminated with a linearly polarized wave, the dipole mode driven by the electric field $\mathrm{E}$, is directly excited on the 8-gap SRR structure. In contrast, the quadrupole dark mode is excited through near field coupling effect. As in ref [7], the combination of the two sub-atoms leads to a transmission band that corresponds to an EIT-like phenomenon.

In order to obtain a final structure with four-fold symmetry, we consider the metamaterial made of an array of multi-gap split ring resonators (MSRRs) with four gaps of equal sizes. The schematic view of the transformation to the proposed metamaterial is presented in Figure 2a. Here we consider an array of MSRR with a lattice constant of $10 \mu \mathrm{m}$. The proposed metamaterial is a planar thin film material with four-fold symmetry that is polarization independent. The transmission and reflection spectra of the structure without the substrate are presented in Figure 2b. Apart from a slight frequency shift, we obtain a response very similar to the one calculated for the 8-gap SRR. We identify the broadband first resonance followed by the sharp resonance. The surface current distributions and the radiation pattern of the MSRR at these two first resonances are presented in Figure 2c. The currents in both resonances show perfect symmetry with respect to the vertical mirror line (parallel to the incident electric field) of the MSRR. The radiation patterns of the MSRR are calculated by considering the surface currents of the modes in a dipolar model [16]. At the first resonance, a dipolar radiation is obtained. It confirms that the first resonance corresponds to the first dipole mode. The currents generated by the first mode were not considered in the calculation of the radiation pattern of the higher order mode. A quadrupolar radiation is observed at the second resonance. This quadrupole appears to be a non-radiative (dark) mode that is usually of high quality factor $(\mathrm{Q} 200)$ as can be observed on the transmission spectrum of Figure $2 \mathrm{~b}$. When illuminated with a linearly polarized wave, the dipole mode driven by the electric field E, is directly excited on the MSRR structure. In contrast, the quadrupole dark mode is excited through near field coupling effect. This bandpass observed around $30 \mathrm{THz}$ in the transmission spectrum of the MSRR is due to Fano interference caused by the interaction of the dark quadrupolar mode with the bright dipolar mode [17,18]. This array of MSRRs achieves an EIT-like phenomenon on the transmission band between the two first modes. 

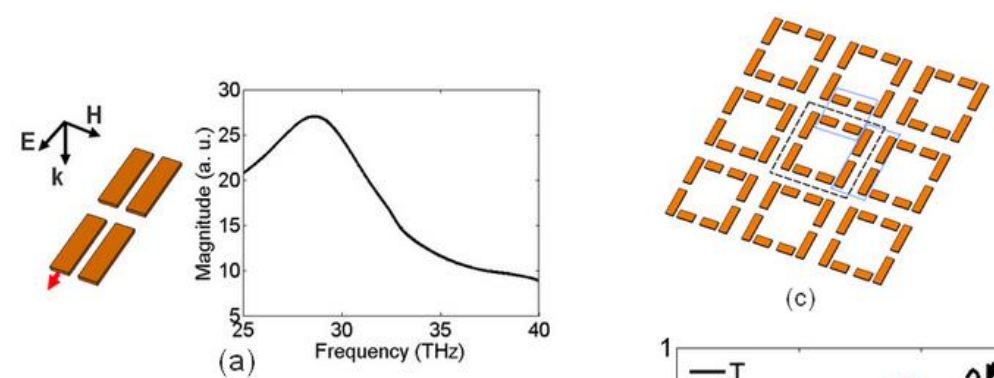

(c)

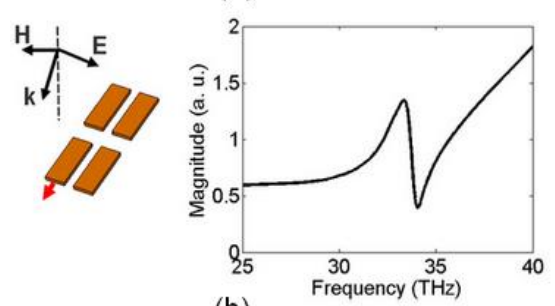

(b)

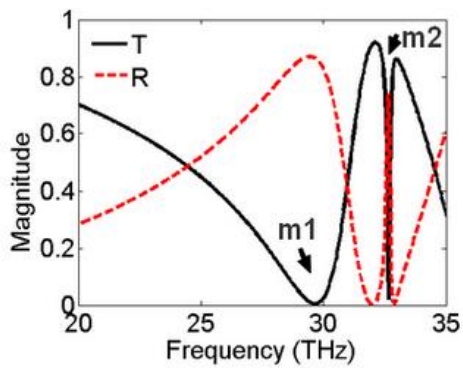

(d)

Figure 1. (a) A pair of two parallel metallic stripes under a normal incidence (left) and the spectral response of an electric probe (red arrow) placed near from the center of the end facet of one metallic stripe (right). (b) A pair of two parallel metallic stripes under an oblique incidence (left) and the spectral response of an electric probe indicated by a red arrow (right). (c) Schematic view of the metamaterial composed of an array of 8-gap split ring resonators (SRRs). (d) Transmission and reflection spectra of the 8-gap SRR.

In order to take a closer look to the properties of the MSRR on the obtained transmission band, we have calculated the effective index of the medium $(n)$ from the obtained transmission and reflection coefficients using the parameter retrieval procedure developed in Ref. [19]. The group index $\left(n_{g}\right)$ of the structure is then calculated from the effective index:

$$
n_{g}=n+f \frac{\partial n}{\partial f}
$$

where $f$ is the frequency. The results are plotted in Figure 3. Group index higher than 100 is obtained near $30 \mathrm{THz}$ in this configuration. Furthermore, the variation of the imaginary part of the effective index shows that the absorption is reduced at the transmission peak and remains low inside the frequency region of interest. Obviously, the presence of a high order resonance next to the fundamental mode produces the EIT-like effect [13].

To investigate the influence of a silicon substrate, the responses of the MSRR-array deposited on a $1-\mu \mathrm{m}$-thick substrate are calculated. The transmission and reflection spectra are presented in Figs 4a. The two first resonances are clearly observed in this configuration. The addition of a high permittivity substrate induces a shift of these two resonances to lower frequencies [20,21]. Furthermore, the transmission coefficient magnitude decreases due to the impedance mismatch caused by the high permittivity of the substrate. Figure $4 \mathrm{~b}$ shows the imaginary part of the index of refraction and the group index of the MSRR deposited on the silicon substrate. The group index of the planar metamaterial becomes larger than 200, whereas $\operatorname{Im}(n)$ is still very low in the EIT-like transmission band. The structure provides a drastic decrease in the group velocity with simultaneously a low absorption. 


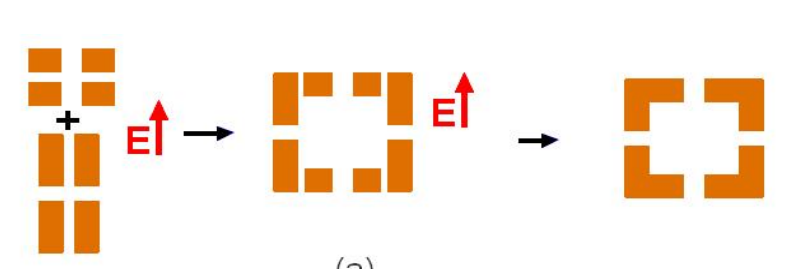

(a)
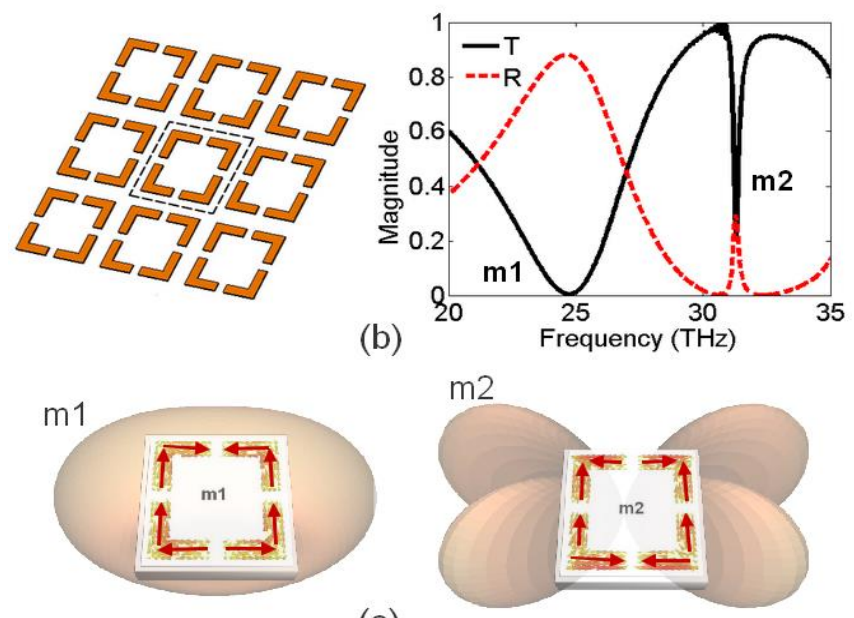

(c)

Figure 2. (a) The transformation from an 8-gap-multi-gap split ring resonator (MSRR) and its corresponding equivalent resonators under a linearly polarized incident wave to the 4-gap MSRR with four-fold symmetry. (b) Schematic view of the metamaterial composed of an array of metallic MSRRs (left) and Transmission and reflection spectra of the MSRR (right). (c) Cartographies of the surface current and the radiation patterns of the MSRR at the two first resonances.

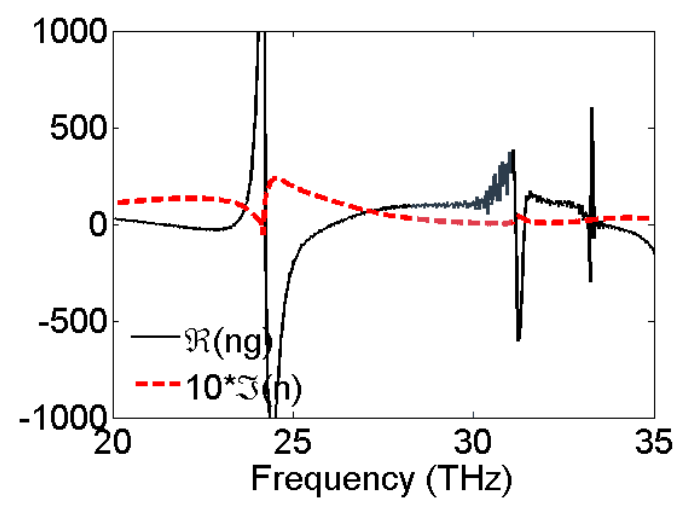

Figure 3. Group index and imaginary part of the effective index of the MSRR structure without substrate. Shaded area shows the domain of EIT. 


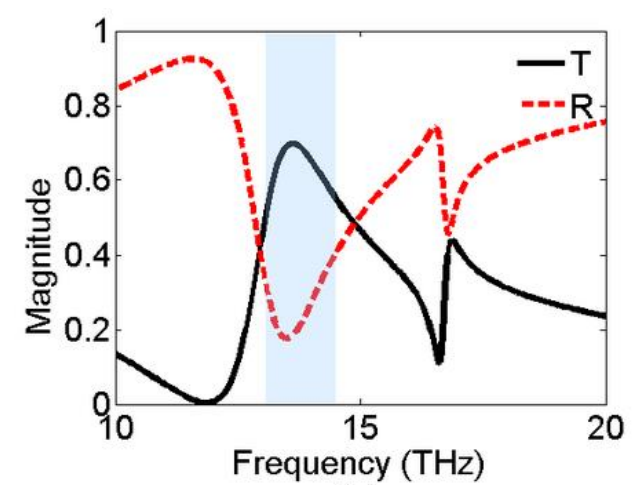

(a)

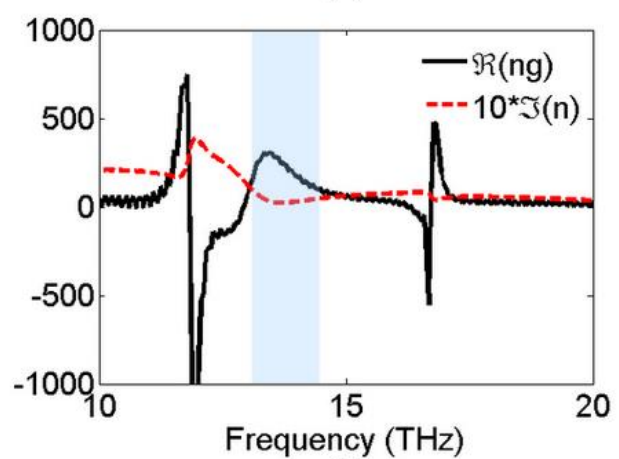

(b)

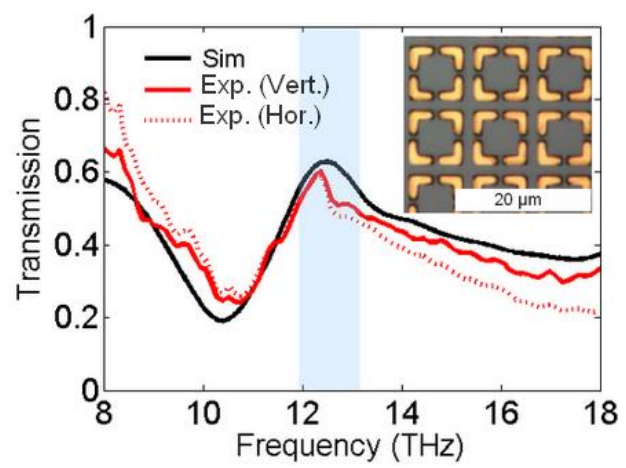

(c)

Figure 4. (a) Transmission and reflection spectra of the metamaterials realized on $1-\mu \mathrm{m}$-thick substrate with $\varepsilon=11.6$. (b) Group index and imaginary part of the effective index of the structure. Shaded area shows the domain of electromagnetically-induced transparency (EIT). (c) Calculated and measured transmission spectra of the metamaterial realized on a semi-infinite substrate. the inset shows the image of the sample realized on a semi-infinite silicon substrate $(\mathrm{h}=280 \mu \mathrm{m})$.

\section{Experimental Results and Discussion}

An experimental sample is realized on a semi-infinite silicon substrate. Using direct writing laser lithography techniques, MSRRs array, with the same dimensions as presented in Figure 1a, is deposited on a n-type $\mathrm{Si}(001)$ substrate [resistivity = 1-10 $\Omega \cdot \mathrm{cm}$ ], 280- $\mu \mathrm{m}$-thick. After being cleaned, the surface of this sample is spin-coated with an adhesion promoter and a positive photoresist (AZ 1518). Then, UV photolithography is used to pattern this resist layer. The illumination step is carried out using a DWL 66FS Heidelberg laser lithography system. The sample is developed and a 
thermal $\mathrm{Au}(80 \mathrm{~nm}) / \mathrm{Cr}(15 \mathrm{~nm})$ deposition is performed. Finally, in order to release the desired design, the excess parts of the resist and metallic layers are removed by an acetone lift-off. Figure $4 \mathrm{c}$ provides an inset that shows an image of the experimental realization of this structure.

The calculated and measured transmission spectra of this structure are presented in Figure 4c. The experimental spectra are obtained for two orthogonal polarizations using a Fourier transform infrared (FTIR) spectrometer. For numerical calculation, we have used $280 \mu \mathrm{m}$-thick substrate and only the first transmitted signal was taken into account to reproduce the effect of an incoherent semi-infinite substrate. Calculated and measured transmission spectra show a good agreement. We clearly identify the narrow transmission band around $12 \mathrm{THz}$ presenting the EIT-like effect as evidenced in Figure 4b. Furthermore, the measured spectra confirm that this metamaterial provide similar response to both vertical and horizontal polarizations of the incident wave.

\section{Conclusions}

In summary, we have shown that EIT-like effect can be obtained in symmetric planar metamaterials. It has been achieved on a specific transmission band in the $\mathrm{THz}$ range, by coherently coupling two consecutive modes of an array of multi-gap split ring resonators deposited on a silicon substrate. The structure we have proposed is planar and shows similar response to both vertical and horizontal polarizations. The MSRR structure may be realized using electron beam lithography to obtain EIT-like behavior in the near infrared range. This type of metamaterial is suitable for slow light applications and enhanced nonlinear effects in the THz and most likely in the infrared and the optical range.

\section{Acknowledgments}

Funding of WOOLLAM IR ellipsometer by the Conseil regional d'Ile-de-France is acknowledged.

This work is supported by LABEX WIFI (Laboratory of Excellence within the French Program "Investments for the Future") under references ANR-10-LABX-24 and ANR-10-IDEX-0001-02 PSL*.

\section{Author Contributions}

A.O. and B. G. initiated this work and conceived the structures and the experiments. L.B realized the prototypes. B.G. and P-R.D. performed the experiments. A.O., B.G. and J. R. analyzed and discussed the results and wrote the manuscript.

\section{Conflict of Interest}

The authors declare no conflict of interest.

\section{References}

1. Harris, S.E. Electromagnetically induced transparency. Phys. Today 1997, 50, 36-42.

2. Boller, K.-J.; Imamolu, A.; Harris, S.E. Observation of electromagnetically induced transparency. Phys. Rev. Lett. 1991, 66, 2593-2596.

3. Maleki, L.; Matsko, A.B.; Savchenkov, A.A.; Ilchenko, V.S. Tunable delay line with interacting whispering-gallery-moderesonators. Opt. Lett. 2004, 29, 626-628. 
4. Lukin, M.D.; Yelin, S.F.; Fleischhauer, M. Entanglement of atomic ensembles by trapping correlated photon states. Phys. Rev. Lett. 2000, 84, 4232-4235.

5. Harris, S.E.; Lene, V.H. Nonlinear optics at low light levels. Phys. Rev. Lett. 1999, 82, 4611-4614.

6. Vassilios, Y.; Emmanuel, P.; Nikolay, V. Electromagnetically induced transparency and slow light in an array of metallic nanoparticles. Phys. Rev. B 2009, 80, 035104.

7. Zhang, S.; Genov, D.A.; Wang, Y.; Liu, M.; Zhang, X. Plasmon-induced transparency in metamaterials. Phys. Rev. Lett. 2008, 101, 047401.

8. Cao, W.; Singh, R.; Zhang, C.; Han, J.; Tonouchi, M.; Zhang, W. Plasmon-induced transparency in metamaterials: Active near field coupling between bright superconducting and dark metallic mode resonators. Appl. Phys. Lett. 2013, 103, 101106.

9. Yan, H.; Low, T.; Guinea, F.; Xia, F.; Avouris, P. Tunable phonon-induced transparency in bilayer graphene nanoribbons. Nano Lett. 2014, 14, 4581-4586.

10. Artar, A.; Yanik, A.A.; Altug, H. Multispectral plasmon induced transparency in coupled meta-atoms. Nano Lett. 2011, 11, 1685-1689.

11. Papasimakis, N.; Fedotov, V.A.; Zheludev, N.I.; Prosvirnin, S.L. Metamaterial analog of electromagnetically induced transparency. Phys. Rev. Lett. 2008, 101, 253903.

12. Lu, Y.; Jin, X.; Zheng, H.; Lee, Y.P.; Rhee, J.Y.; Jang, W.H. Plasmonic electromagnetically-induced transparency in symmetric structures. Opt. Express 2010, 18, 13396-13401.

13. Jin, X.-R.; Lu, Y.; Zheng, H.; Lee, Y.P.; Rhee, J.Y.; Kim, K.W.; Jang, W.H. Plasmonic electromagnetically-induced transparency in metamaterial based on second-order plasmonic resonance. Opt. Commun. 2011, 284, 4766-4768.

14. Chiam, S.-Y.; Singh, R.; Rockstuhl, C.; Lederer, F.; Zhang, W.; Bettiol, A.A. Analogue of electromagnetically induced transparency in a terahertz metamaterial. Phys. Rev. B 2009, 80, 153103.

15. Tassin, P.; Zhang, L.; Koschny, T.; Economou, E.N.; Soukoulis, C.M. Planar designs for electromagneticallyinduced transparency in metamaterials. Opt. Express 2009, 17, 5595-5605.

16. Jouveaud, C.; Ourir, A.; Rosny, J. Surface waves radiation by finite arrays of magnetoelectric resonators. Prog. Electromagn. Res. 2012, 132, 177-198.

17. Zhang, S.; Bao, K.; Halas, N.J.; Xu, H.; Nordlander, P. Substrate-induced fano resonances of a plasmonic nanocube: A route to increased-sensitivity localized surface plasmon resonance sensors revealed. Nano Lett. 2011, 11, 1657-1663.

18. Lu, Y.; Rhee, J.Y.; Jang, W.H.; Lee, Y.P. Active manipulation of plasmonic electromagneticallyinduced transparency based on magnetic plasmon resonance. Opt. Express 2010, 18, 20912-20917.

19. Smith, D.R.; Schultz, S.; Markoš, P.; Soukoulis, C.M. Determination of effective permittivity and permeability of metamaterials from reflection and transmission coefficients. Phys. Rev. B 2002, 65, 195104.

20. Bedeaux, D.; Vlieger, J. A statistical theory for the dielectric properties of thin island films: Application and comparison with experimental results. Thin Solid Films 1983, 102, 265-281. 
21. Lazzari, R.; Stéphane, R.; Ingve, S.; Jacques, J.; Dick, B.; Jan, V. Multipolar plasmon resonances in supported silver particles: The case of $\mathrm{Ag} / \alpha-\mathrm{Al}_{2} \mathrm{O}_{3}(0001)$. Phys. Rev. B 2002, 65, 235424.

(C) 2015 by the authors; licensee MDPI, Basel, Switzerland. This article is an open access article distributed under the terms and conditions of the Creative Commons Attribution license (http://creativecommons.org/licenses/by/4.0/). 